\title{
ON/OFF SOLENOID WITH SENSORLESS POSITION DETECTION
}

\author{
Peter Tappe*, Jürgen Heinzmann, Ralf Raupold \\ Magnet-Schultz GmbH, Allgäuer Str. 30, 87700 Memmingen \\ * Corresponding author: Tel.: +49 8331104 392; E-mail address: peter.tappe@magnet-schultz.de
}

\begin{abstract}
As is generally known, pressure-resistant solenoids for valves are used in hydraulics and pneumatics. These solenoids, for example, consist of a so-called pole tube with an integrated armature and a separate solenoid coil. Suitable displacement measuring systems are used in order to measure the armature's as well as valve spool's position constantly; these are directly mounted to the solenoid. Examples here are Hall Effect sensors or LVDT transducers. In case of lesser requirements, it is also possible to measure the valve spool's position directly and in simplified manner. Here, partially standard proximity sensors are employed, which use the valve spool directly as measuring object. In these cases, however, only discrete position measurement is possible. A constant displacement measurement is not necessary. By means of the sensorless position-control developed by MSM, the armature's position can be executed directly by analyzing the solenoid coil, partly replacing the usual sensors, under adapted basic conditions.
\end{abstract}

Keywords: Sensor, LVDT, Hall-Effekt, sensorless

\section{INTRODUCTION}

The on/off solenoids usually used in hydraulic valves have a comparably thick-wall pole tube, with a non-magnetic zone integrated. The armature inside such pole tube creates the necessary linear force in order to achieve the valve's full function. Such constructions are also used for sophisticated pneumatic valves.

In many valve applications, the valve spool's position needs to be measured. This is usually achieved by separate sensors, whose measuring elements are connected to the valve spool or, at least, to the solenoid armature. The functionality principle of such sensors is, for example, based on the LVDT principle or uses Hall Effect sensors.

Future developments may promote using sensor information. As application areas, the keywords Industry 4.0 or Predictive Maintenance can be mentioned.

However, regarding such applications, there is a lesser requirement for the position detection. For example, it might be sufficient to sense the currentless solenoid armature's final position only. The valve spool's position determined here displays the valve's cut-off state and so the unpressurized working port.
If, in such cases, sensing the armature's final position is sufficient, the procedure developed by MSM for sensorless position detection may be used.

The basics of the solenoid coil's direct usage to determine the armature's position are described in this article.

\section{CONSTANT PATH SENSORS}

Principally, sensing of final position is also possible with constantly operating transducers and has been frequently applied in practice. The LVDT principle is widespread. As is generally known, a non-permanent core is moved within a 3 chamber coil system. The position has an impact on the current induced into the secondary coils by a primary coil, so that position detection is possible.

For an exemplary measuring range of $+/-4$ $\mathrm{mm}$, a linearity of $+/-1 \%$ within a temperature range of -25 to $+75^{\circ} \mathrm{C}$ can be achieved. 


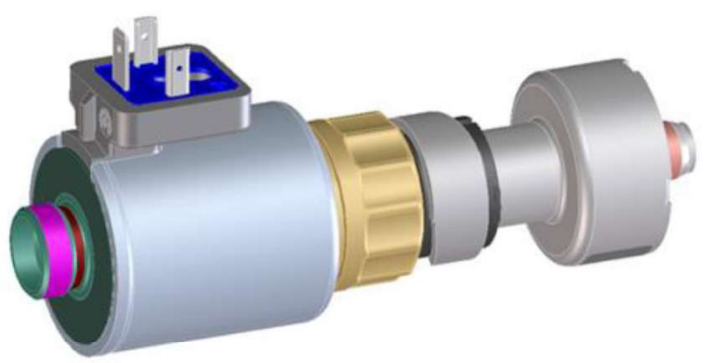

Figure 1: Solenoid with path sensor (LVDT measuring principle)

Even in hydraulics and pneumatics, transducers that use Hall Effect sensors are widespread. These sensors measure the magnetic field of a permanent magnet fastened with a measuring object. Such sensors may also be used as autonomous sensors, independent of actuators.

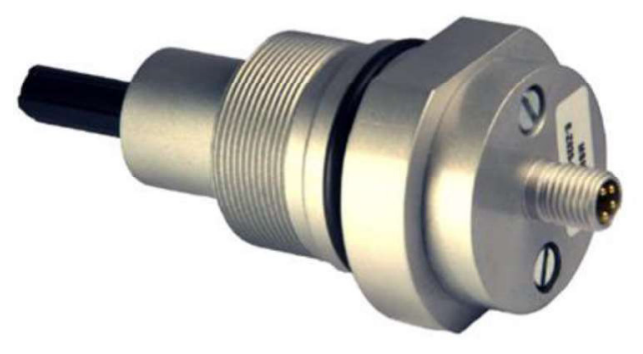

Figure 2: Solenoid with position sensor (hall sensor)

With this method of action, for example, a linearity of $+/-1,5 \%$ within a temperature range of -25 to $+105^{\circ} \mathrm{C}$ and a measuring range of $+/-6 \mathrm{~mm}$ can be achieved.

\subsection{Position control}

Due to cost advantages, the hall sensor principle is increasingly important for position control. Position control is also based on a constantly measurable physical principle, however, only final positions are issued.

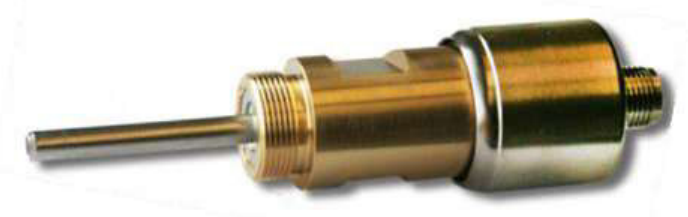

Figure 3: $\quad$ Solenoid with position control (hall sensor)
When a position control detects 2 positions, for example, the following data is achieved: measuring range $+/-8.5 \mathrm{~mm}$, output voltage $>7$ $\mathrm{V}$ and/or $<1.5 \mathrm{~V}$ within a temperature range of 40 to $100^{\circ} \mathrm{C}$

\section{SENSORLESS POSITION CONTROL}

\subsection{Inductance of a solenoid coil}

On contemplating such an air coil, its inductance is ideal and constant. However, a coil within the solenoid's iron circle is not constant when taking into account the progression versus the armature's position.

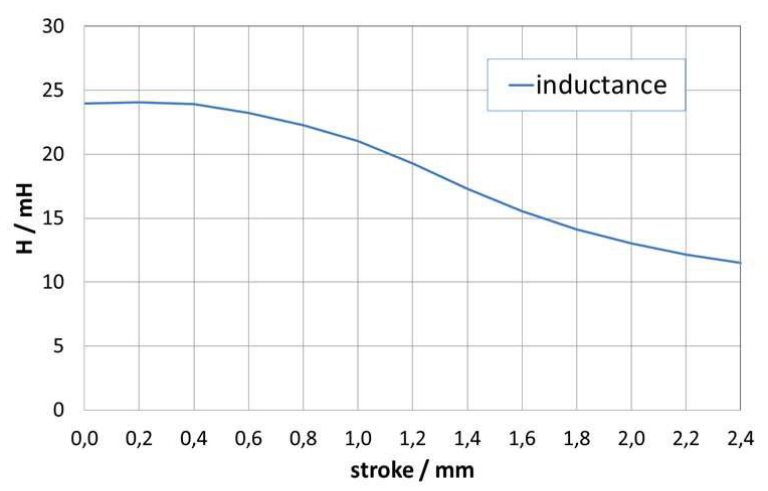

Figure 4: Inductance of a copper coil within an iron circle

The diagram of the typical inductance progression points out that it is apparently attractive to make use of these characteristics in order to detect the armature's position.

It must be emphasized, however, that such a progression can only be achieved under quasistatic basic conditions. In order to measure such an inductance progression, the solenoid's armature is analyzed in discrete measuring positions, for example. In case of energized solenoids in real applications, induced voltages are created within the magnetic field by the armature's movement. So the impact on coil current due to inductance is not only changed solely by position but also by the armature's dynamic movement itself, making it very difficult or even impossible to analyze constantly when considering usual requirements for the optimal functioning of solenoids in valves. Such requirements include, for example, the accuracy of a constant path signal or the optimal design of the solenoid's circle with regard to efficiency. 
This is the reason why the method presented here concentrates on the analysis of the currentless solenoid coil's idle state.

\subsection{Analysis principle}

When taking power off an energized copper coil, a typical voltage peak is created. The voltage's height is usually limited by TVS diodes. Principally, the transient progression of the voltage is influenced by the coil's inductance.

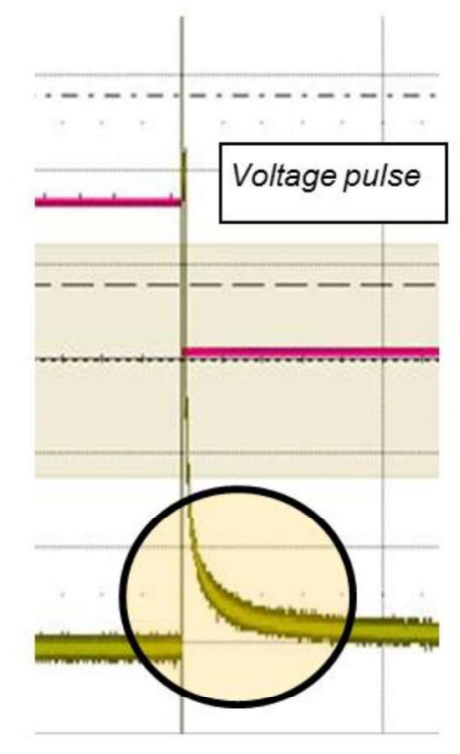

Figure 5: voltage pulse during switch-off

Since now, in turn, the inductance is dependent on the armature's position, an analyzable criteria regarding the armature's position is available. Our MSM patented procedure includes analysis of the duration from switch-off to a defined voltage level's underrun. Duration is measured by evaluating a timer.

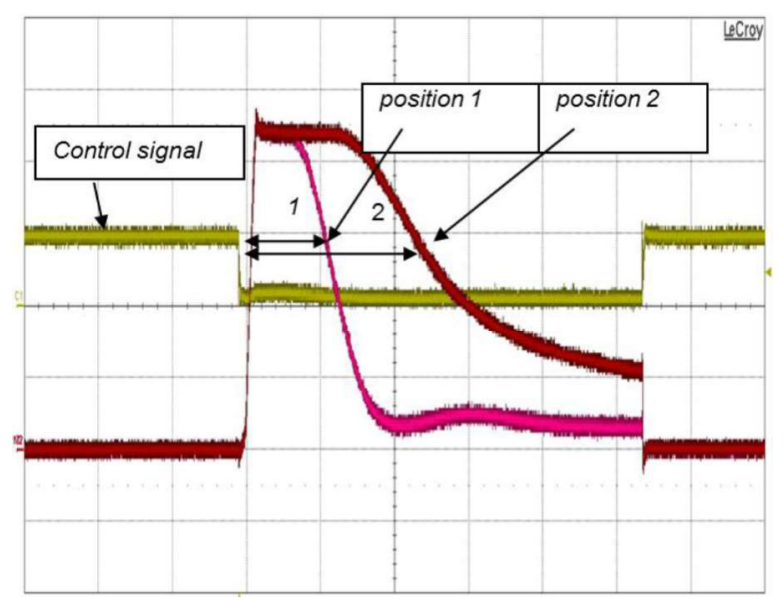

Figure 6: voltage pulse of 2 armature positions
For an application's usage, the solenoid coil is energized by regular measuring pulses so that enough positon information is created. The respective pulse during energizing does not lead to the armature's movement when suitably dimensioning the overall function.

In order to complete the description of the measuring principle, the following situation explains when a solenoid is energized. In case a solenoid coil is regulated by the usual PWM operation, particularly in shift valves, cyclical switch-offs are created. These may be analyzed as well. An elaborate harmonization is needed for such switch-off practicability without negative impact on the valve's function, so this operation situation is not part of this report.

\section{FUNCTIONAL EXAMPLE}

The following Picture demonstrates the integration of a sensorless position detection in a bi-stable shift valve. This solenoid is a pulling version which may be operated in bi-stable manner by using a permanent solenoid. Due to the bi-stable active principle, position may be ideally analyzed in currentless state.

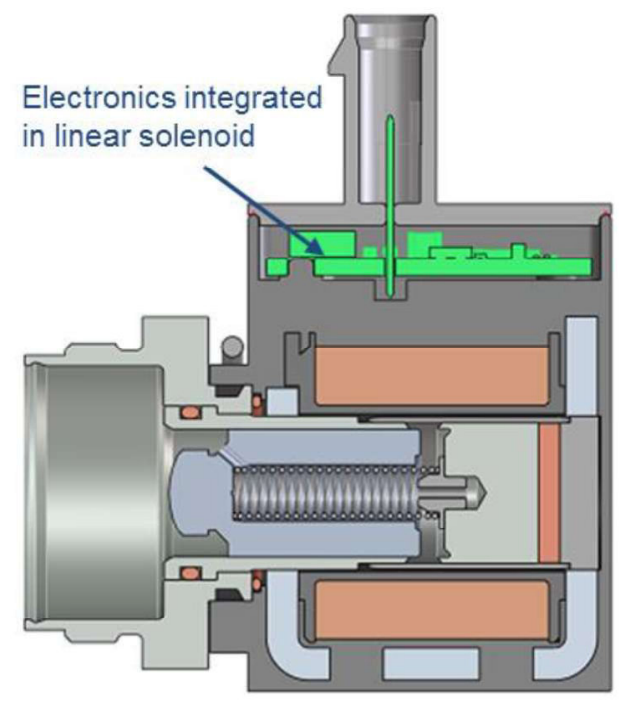

Figure 7: integration of sensorless position detection in a bi-stable shift valve

The solenoid is completed as a shift valve by the customer. The electronic systems include the following functions:

- Energizing by $\mathrm{H}$ bridge

- Controlled by LIN/CAN bus

- Supply voltage 9-18 V DC

- Peak current 5 A 
- - Temperature range $-40 \ldots 125^{\circ} \mathrm{C}$

- - Dimension of printed board $31 \times 31 \mathrm{~mm}^{2}$ The magnetic stroke of the shift valve used here is 0 to $3.0 \mathrm{~mm}$. Within this range, the sensorless position detection can issue a range of $0.1 \ldots 0.7$ $\mathrm{mm}$ (typically $0.45 \mathrm{~mm}$ ) for the energized state. Regarding the off-peak state, an armature position of $1.3 \mathrm{~mm}$ and larger (typically $1.41 \mathrm{~mm}$ ) can be detected.

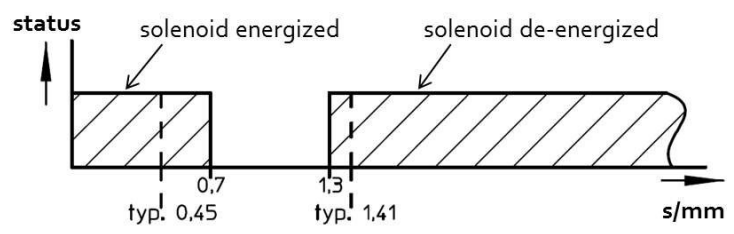

Figure 8: position detection

The switch point precision was achieved under the following basic conditions:

- Ambient temperature $-40 \ldots 125^{\circ} \mathrm{C}$

- Supply voltage 9...16 V

- Use of an averaged temperature compensation

Under the above-mentioned basic conditions, the measurement precision as indicated is achieved. At the same time, the disadvantages of the analysis of the inductance caused by physical conditions are visible. In case of a large stroke, the typical inductance progression flattens increasingly, with the measuring principle's resolution deteriorating due to physical conditions in case of large strokes.

By means of an individual programming of temperature compensation, the measuring quality, however, can be improved considerably.

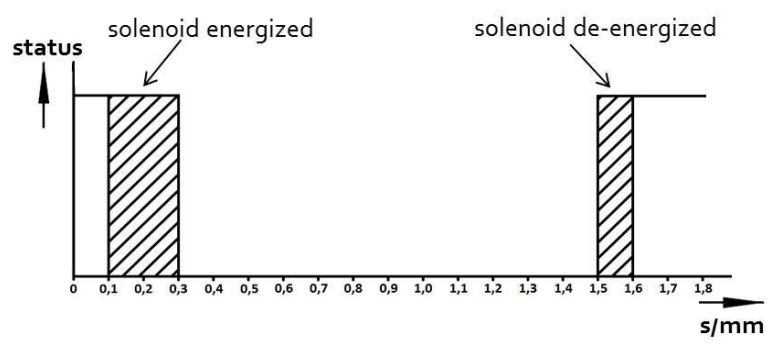

Figure 9: Optimized position detection

\subsection{Application}

The next picture shows the usage of two on/off solenoids for a hydraulic valve. In order to achieve the valve spool's position detection, the use of ordinary sensors is possible. If the sensors mounted on solenoids are replaced by sensorless position control, a subsequent functional detection might be possible.

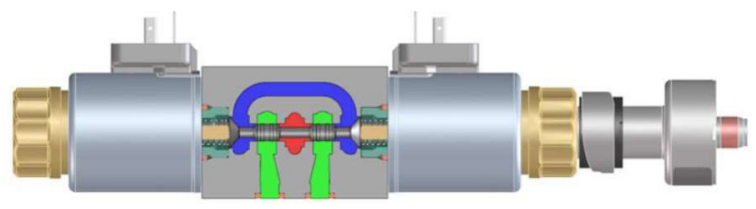

Figure 10: Valve with 2 on/off solenoids including sensor

The uncritical operation state is the valve spool's centered middle position. In this state, working ports are without pressure. The valve spool's deficient eccentric position inevitably moves one of the solenoid's armature from its operation position towards idle stroke. If the armature position is now being monitored, a hydraulic malfunction of such type can be detected.

For a typical solenoid, the principle signal quality's progression (number of metering pulses) of the sensorless positon detection can be recognized in the next picture. It can be seen that due to the physical characteristics of the inductance, partial areas are created - these may be analyzed as well.

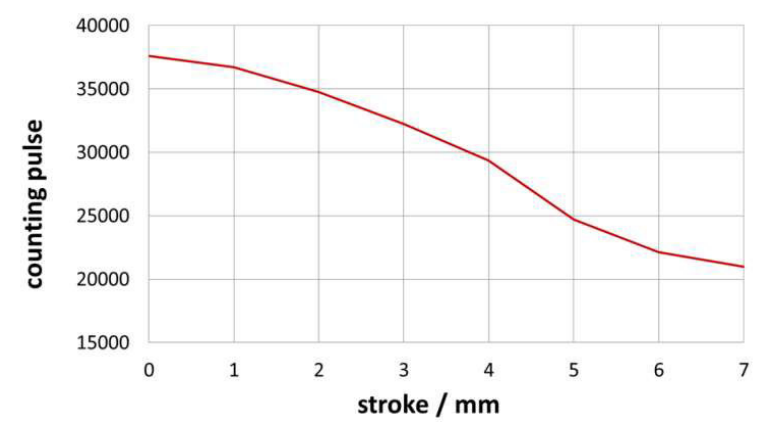

Figure 11: signal quality above stroke area

If both solenoid coils are differentially analyzed, the signal's resolution can, under ideal basic conditions, be improved.

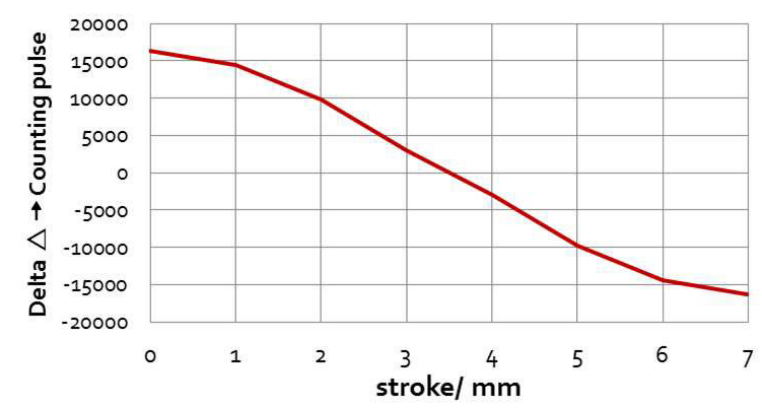

Figure 12: Signal quality above stroke area in case 2 solenoid coils are used 


\section{BASIC CONDITIONS}

A solenoid's inductance progression is the basis for the position determination by means of sensorless position detection. This progression can certainly not be optimized independent of force/stroke behavior. The arrangement of magnetizable areas of the iron circle and the operation of the iron components in saturation usually aim at an ideal force/stroke behavior for the application in valves.

For a reasonable usage of the sensorless position detection, an optimal configuration of the inductance at the expense of the force/stroke behaviour is necessary. This is the reason why the application of a sensorless position detection is actually not possible without amending the valve's dimensioning and potentially limiting the hydraulic parameters.

\section{CONCLUSION}

In this report, the inductance characteristics of a solenoid are used to create a sensorless position detection. When the armature of an energized solenoid moves, the current progression is influenced by self-induced voltages due to the armature's movement. So the usage of a sensor principle only makes sense in a case where the armature is in idle state, for example, after a switching procedure's completion.

The sensor principle measures the progression of the switch-off excess voltage which is typically created during switch-offs. So, if measuring pulses are applied onto the solenoid coil, measurements are possible in sufficient chronology.

In the report, valves which are, amongst others, operated by two solenoids, have been examined. In this configuration, one of the solenoids is used as sensor element while the opposite solenoid is energized in order to operate the valve.

Usually, the force/stroke behavior is adjusted ideally to the valve's function. The inductance's characteristic diagram that is created here depends thereon and can certainly not be optimized independently. So it is necessary to optimize the whole valve comprehensively in order to be able to make reasonable use of the inductance progression. The valve, together with the solenoid, must be configured virtually in "sensor conforming" manner.
With such valves and the respective solenoids, replacing traditional sensors is possible under fixed basic conditions.

\section{REFERENCES}

1) Jürgen Heinzmann: Sensorless Position Detection for Electromagnetic Actuators, IFK-Proceedings 8. IFK 2012 